\title{
CORRECTION
}

\section{Correction to: Catheter retention as a consequence rather than a cause of unfavorable outcome in candidemia}

\author{
Lauro Damonti ${ }^{1}$, Véronique Erard ${ }^{1,2}$, Jorge Garbino ${ }^{3}$, Jacques Schrenzel ${ }^{4}$, Stefan Zimmerli ${ }^{5}$, \\ Konrad Mühlethaler ${ }^{5}$, Alexander Imhof ${ }^{6,8}$, Reinhard Zbinden ${ }^{7}$, Jan Fehr ${ }^{8}$, Katia Boggian $^{9}$, Thomas Bruderer ${ }^{10}$, \\ Ursula Flückiger ${ }^{11,14}$, Reno Frei ${ }^{12}$, Christina Orasch ${ }^{1,13,14}$, Anna Conen ${ }^{14,15}$, Nina Khanna ${ }^{14}$, \\ Thomas Bregenzer ${ }^{15,16}$, Jacques Bille ${ }^{17}$, Frédéric Lamoth ${ }^{1,17}$, Oscar Marchetti ${ }^{1}$, $^{*}$, Pierre-Yves Bochud ${ }^{1 *}$ (D) \\ and Fungal Infection Network of Switzerland (FUNGINOS)
}

(C) 2018 Springer-Verlag GmbH Germany, part of Springer Nature and ESICM

\section{Correction to: Intensive Care Med (2017) 43:935-939 https://doi.org/10.1007/s00134-017-4737-9}

In the original publication the members of the FUNGINOS network were provided in such a way that they could not be indexed as collaborators on PubMed. The publisher apologizes for this error and is pleased to list the members of the network here:

\footnotetext{
*Correspondence: Oscar.Marchetti@chuv.ch;

Pierre-Yves.Bochud@chuv.ch

${ }^{1}$ Infectious Diseases Service, Department of Medicine, Lausanne University Hospital, Rue du Bugnon 46, CH-1011 Lausanne, Switzerland

Full author information is available at the end of the article
}

\section{Springer}




\section{Clinical Investigators (Infectious Diseases specialists)}

\begin{tabular}{|c|c|c|c|c|}
\hline First name & Last name & Affiliation & City & Country \\
\hline Thomas & Bregenzer & Kantonsspital & Aarau & Switzerland \\
\hline \multirow[t]{2}{*}{ Anna } & \multirow[t]{2}{*}{ Conen } & Kantonsspital & Aarau & Switzerland \\
\hline & & Basel University Hospital & Basel & Switzerland \\
\hline Ursula & Flückiger & Basel University Hospital & Basel & Switzerland \\
\hline Nina & Khanna & Basel University Hospital & Basel & Switzerland \\
\hline \multirow[t]{2}{*}{ Christina } & Orasch & Basel University Hospital & Basel & Switzerland \\
\hline & & Lausanne University Hospital & Lausanne & Switzerland \\
\hline Ulrich & Heininger & Universitätskinderspital & Basel & Switzerland \\
\hline Mario & Franciolli & Ospedale San Giovanni, Ente Ospedaliero Cantonale & Bellinzona & Switzerland \\
\hline Stefan & Zimmerli & Bern University Hospital & Bern & Switzerland \\
\hline \multirow[t]{2}{*}{ Madeleine } & \multirow[t]{2}{*}{ Rothen } & Spitalzentrum & Biel & Switzerland \\
\hline & & Kantonsspital & Bruderholz & Switzerland \\
\hline Claudine & Zellweger & Spitalzentrum & Biel & Switzerland \\
\hline Philipp & Tarr & Kantonsspital & Bruderholz & Switzerland \\
\hline Felix & Fleisch & Kantonsspital & Chur & Switzerland \\
\hline Christian & Chuard & Hôpital Cantonal & Fribourg & Switzerland \\
\hline Stéphane & Emonet & Geneva University Hospital & Geneva & Switzerland \\
\hline Jorge & Garbino & Geneva University Hospital & Geneva & Switzerland \\
\hline Christian & van Delden & Geneva University Hospital & Geneva & Switzerland \\
\hline Daniel & Genne & Hôpital Communal & La-Chaux-de-Fonds & Switzerland \\
\hline Pierre-Yves & Bochud & Lausanne University Hospital & Lausanne & Switzerland \\
\hline Thierry & Calandra & Lausanne University Hospital & Lausanne & Switzerland \\
\hline \multirow[t]{2}{*}{ Lauro } & \multirow[t]{2}{*}{ Damonti } & Lausanne University Hospital & Lausanne & Switzerland \\
\hline & & Bern University Hospital & Bern & Switzerland \\
\hline \multirow[t]{2}{*}{ Véronique } & \multirow[t]{2}{*}{ Erard } & Lausanne University Hospital & Lausanne & Switzerland \\
\hline & & Hôpital Cantonal & Fribourg & Switzerland \\
\hline Frédéric & Lamoth & Lausanne University Hospital & Lausanne & Switzerland \\
\hline Oscar & Marchetti & Lausanne University Hospital & Lausanne & Switzerland \\
\hline Jean-Philippe & Chave & Clinique Bois-Cerf, Clinique Cécil, and Clinique La Source & Lausanne & Switzerland \\
\hline Peter & Graber & Kantonsspital & Liestal & Switzerland \\
\hline Rita & Monotti & Ospedale Regionale La Carità, Ente Ospedaliero Cantonale & Locarno & Switzerland \\
\hline Enos & Bernasconi & Ospedale Civico, Ente Ospedaliero Cantonale & Lugano & Switzerland \\
\hline Marco & Rossi & Kantonsspital & Luzern & Switzerland \\
\hline Martin & Krause & Kantonsspital & Münsterlingen & Switzerland \\
\hline Rein-Jan & Piso & Kantonsspital & Olten & Switzerland \\
\hline Frank & Bally & Institut Central des Hôpitaux Valaisans & Sion & Switzerland \\
\hline Nicolas & Troillet & Institut Central des Hôpitaux Valaisans & Sion & Switzerland \\
\hline Katia & Boggian & Kantonsspital & Sankt Gallen & Switzerland \\
\hline \multirow[t]{2}{*}{ Jacques } & \multirow[t]{2}{*}{ Gubler } & Kantonsspital & Winterthur & Switzerland \\
\hline & & Stadtspital Triemli & Zürich & Switzerland \\
\hline Jan & Fehr & Zürich University Hospital & Zürich & Switzerland \\
\hline Alexander & Imhof & Zürich University Hospital & Zürich & Switzerland \\
\hline Christian & Ruef & Zürich University Hospital & Zürich & Switzerland \\
\hline \multirow[t]{2}{*}{ Gerhard } & \multirow[t]{2}{*}{ Eich } & Stadtspital Triemli & Zürich & Switzerland \\
\hline & & Kantonsspital & Winterthur & Switzerland \\
\hline Christoph & Berger & Universitätskinderspital & Zürich & Switzerland \\
\hline
\end{tabular}


Laboratory Investigators (Microbiologists)

\begin{tabular}{|c|c|c|c|c|}
\hline First name & Last name & Affiliation & City & Country \\
\hline Hans & Fankhauser & Kantonsspital & Aarau & Switzerland \\
\hline IVo & Heinzer & Kantonsspital & Aarau & Switzerland \\
\hline Reno & Frei & Basel University Hospital & Basel & Switzerland \\
\hline Roland & Hertel & Universitätskinderspital & Basel & Switzerland \\
\hline Marisa & Dolina & Istituto Cantonale di Microbiologia & Bellinzona & Switzerland \\
\hline Orlando & Petrini & Istituto Cantonale di Microbiologia & Bellinzona & Switzerland \\
\hline Olivier & Dubuis & Viollier Microbiology Laboratories & Bienne & Switzerland \\
\hline Konrad & Mühlethaler & Bern University Hospital & Bern & Switzerland \\
\hline \multirow[t]{2}{*}{ Suzanne } & \multirow[t]{2}{*}{ Graf } & Kantonsspital & Bruderholz & Switzerland \\
\hline & & Kantonsspital & Liestal & Switzerland \\
\hline Martin & Risch & Kantonsspital & Chur & Switzerland \\
\hline Eva & Ritzler & Kantonsspital & Chur & Switzerland \\
\hline Dominique & Fracheboud & Hôpital Cantonal & Fribourg & Switzerland \\
\hline Peter & Rohner & Geneva University Hospital & Geneva & Switzerland \\
\hline Jacques & Schrenzel & Geneva University Hospital & Geneva & Switzerland \\
\hline Reto & Lienhardt & Hôpital Communal & La-Chaux-de-Fonds & Switzerland \\
\hline Jacques & Bille & Lausanne University Hospital & Lausanne & Switzerland \\
\hline Frédéric & Lamoth & Lausanne University Hospital & Lausanne & Switzerland \\
\hline Corinne & Andreutti-Zaugg & Clinique La Source & Lausanne & Switzerland \\
\hline Alberto & Gallusser & Clinique La Source & Lausanne & Switzerland \\
\hline Gaby & Pfyffer & Kantonsspital & Luzern & Switzerland \\
\hline Urs & Schibli & Kantonsspital & Olten & Switzerland \\
\hline Lysiane & Tissière & Institut Central des Hôpitaux Valaisans & Sion & Switzerland \\
\hline Thomas & Bruderer & Kantonsspital & Sankt Gallen & Switzerland \\
\hline Detlev & Schultze & Kantonsspital & Sankt Gallen & Switzerland \\
\hline Reinhard & Zbinden & Zürich University Hospital & Zürich & Switzerland \\
\hline
\end{tabular}

\section{Author details}

${ }^{1}$ Infectious Diseases Service, Department of Medicine, Lausanne University Hospital, Rue du Bugnon 46, CH-1011 Lausanne, Switzerland. ${ }^{2}$ Department of Medicine, HFR-Fribourg Cantonal Hospital, Fribourg, Switzerland. ${ }^{3}$ Infectious Diseases Service, Department of Medical Specialties, Geneva University Hospitals, Geneva, Switzerland. ${ }^{4}$ Bacteriology Laboratory, Service of Laboratory Medicine, Department of Genetics and Laboratory Medicine, Geneva University Hospitals, Geneva, Switzerland. ${ }^{5}$ Institute for Infectious Diseases, University of Bern, Bern, Switzerland. ${ }^{6}$ Department of Medicine, Oberaargau Hospital, Langenthal, Switzerland. ${ }^{7}$ Laboratory of Microbiological Diagnostics, Institute of Medical Microbiology, University of Zürich, Zürich, Switzerland.

${ }^{8}$ Division of Infectious Diseases and Hospital Epidemiology, Zürich University Hospital, Zürich, Switzerland. ${ }^{9}$ Division of Infectious Diseases and Hospital Epidemiology, Cantonal Hospital, Sankt Gallen, Switzerland. ${ }^{10}$ Department of Bacteriology, Mycology and Parasitology, Center of Laboratory Medicine,
Cantonal Hospital, Sankt Gallen, Switzerland. ${ }^{11}$ Internal Medicine and Infectious Diseases, Hirslanden Klinik, Aarau, Switzerland. ${ }^{12}$ Laboratory Medicine, Division of Clinical Microbiology, Basel University Hospital, Basel, Switzerland.

${ }^{13}$ Internal Medicine and Infectious Diseases, Hirslanden Klinik, St. Anna, Lucerne, Switzerland. ${ }^{14}$ Division of Infectious Diseases and Hospital Epidemiology, Basel University Hospital, Basel, Switzerland. ${ }^{15}$ Division of Infectious Diseases and Hospital Hygiene, Cantonal Hospital, Aarau, Switzerland. ${ }^{16}$ Clinics for Internal Medicine, Hospital Lachen AG, Lachen, Switzerland. ${ }^{17}$ Institute of Microbiology, Department of Laboratories, Lausanne University Hospital, Lausanne, Switzerland. ${ }^{18}$ Department of Medicine, Ensemble Hospitalier de la Côte, Morges, Switzerland.

Published online: 20 February 2018 